\title{
MORFOMETRIA INTESTINAL E QUALIDADE ÓSSEA DE CODORNAS JAPONESAS ALIMENTADAS POR UM PERÍODO PROLONGADO COM COPRODUTOS DO ÓLEO DE SOJA
}

\author{
(Intestinal morphometry and bone quality of Japanese quails fed for a prolonged \\ period with soybean oil by-products)
}

Caroline Bavaresco, Ana Paula Nunes, Juliana Forgiarini, Débora Aline Alves, Eduardo Gonçalves Xavier, Débora Cristina Nichelle Lopes, Victor Fernando Büttow Roll.

Universidade Federal de Pelotas, RS, Brasil.

1'Autor para correspondência: carolinebavaresco@hotmail.com

RESUMO - O objetivo do presente estudo foi avaliar os efeitos do uso prolongado de lecitina e óleo ácido de soja sobre a histologia duodenal e qualidade óssea de codornas japonesas na fase de postura. Foram utilizados 192 aves com 54 dias de idade as quais foram alimentadas durante 168 dias (metade da sua vida produtiva) com dietas contendo óleo degomado de soja (ODS) ou óleo ácido de soja (OAS) associadas ou não com lecitina bruta de soja (LEC), totalizando 8 tratamentos descritos à seguir: T1 - dieta com 4\% OAS; T2 - dieta com 4\% OAS e 1\% LEC; T3 dieta com 8\% OAS; T4 - dieta com 8\% OAS e 1\% LEC; T5 - dieta com 4\% ODS; T6 - dieta com 4\% ODS e 1\% LEC; T7 - dieta com 8\% ODS; T8 - dieta com 8\% ODS e $1 \%$ LEC. Ao final do período experimental foram coletadas amostras do duodeno para morfometria de vilos e criptas, e as tíbias para as análises de força, rigidez, flexibilidade e cinzas totais. Foi verificado interação dupla significativa entre tipo de óleo e lecitina para profundidade de cripta $(P=0,04)$. Codornas alimentadas com dietas contendo OAS na presença de LEC apresentaram significativamente menor profundidade de cripta em relação as aves alimentas apenas com OAS. No entanto, quando as aves foram alimentadas com ODS essas diferenças devido a presença de LEC não foram significativas. O nível mais alto de óleo (8\%) na dieta e a lecitina $(1 \%)$ reduziram o conteúdo total de cinzas nas tíbias $(P<0,05)$. Conclui-se que a associação entre lecitina e o óleo ácido de soja foi positiva para a manutenção do tecido intestinal, mas não afetou a qualidade óssea das aves.

Palavras-chave: Coturnix coturnix japonica; lecitina; minerais; óleo ácido de soja; vilos.

\begin{abstract}
The objective of the present study was to evaluate the effects of prolonged use of lecithin and soybean oil on the duodenal histology and bone quality of Japanese quails in laying phase. A hundred and ninety-two birds with 54 days of age were fed for 168 days (half of their productive life) with diets containing degummed soybean oil (DSO) or acidulated soybean soapstock oil (ASS) in association or not with raw soybean lecithin (LEC), totaling eight treatments described below: T1 - diet with 4\% ASS; T2 - diet with 4\% ASS and 1\% LEC; T3 diet with $8 \%$ ASS; T4 - diet with $8 \%$ ASS and $1 \%$ LEC; T5 - diet with 4\% DSO; T6 diet with $4 \%$ DSO and 1\% LEC; T7 - diet with 8\% DSO; T8 - diet with $8 \%$ DSO and $1 \%$ LEC. At the end of the experimental period, duodenum samples were collected for morphometry of villus and crypts, and tibias were using for analysis of strength, rigidity, flexibility and total ashes. Significant double interaction between oil type and lecithin was found for crypt depth $(P=0.04)$. Quails fed diets containing ASS in the presence of LEC showed significantly lower crypt depth compared to birds fed with
\end{abstract}


ASS only. However, when birds were fed DSO these differences due to the presence of LEC did not significant. The highest level of oil (8\%) and the lecithin $(1 \%)$ reduced the total ash tibia content $(P<0.05)$. It was concluded that the association between lecithin and acidulated soybean soapstock oil was positive for the maintenance of the intestinal tissue but did not affect the bone quality of the birds.

Keywords - acidulated soybean soapstock oil; Coturnix coturnix japonica; lecithin; minerals, villus. 


\section{INTRODUÇÃO}

Diferentes ingredientes podem ser utilizados para fornecer energia na dieta de aves, desde fontes vegetais até subprodutos da indústria, sem que afete negativamente 0 desempenho dos animais (Firman et al., 2008). A suplementação das dietas com óleos ou gorduras trás diversos benefícios aos animais além do fornecimento de energia, como a melhora da digestibilidade dos nutrientes, redução da pulverulência das rações, fontes de ácidos graxos essenciais, entre outros (Braga e Baião, 2001). No entanto, a qualidade da fonte lipídica é de extrema importância, pois é um fator que limita a absorção dos componentes dos óleos. Um exemplo disso é quando as fontes lipídicas possuem altas quantidades de ácidos graxos livres (AGL), acima de $60 \%$, como é o caso do óleo ácido de soja (OAS). Segundo Leeson e Summers (2005) de 50 a $78 \%$ dos lipídios são absorvidos na forma de 2monoacilglicerol, e esse fato explicaria o atraso no crescimento de aves quando alimentadas com elevados índices de AGL, já que afeta o aproveitamento das gorduras e de outros nutrientes.

A digestão final do alimento e absorção dos nutrientes ocorre no intestino delgado, onde estão presentes alguns tipos de células responsáveis pela digestão e transporte dos nutrientes, proteção do epitélio e produção de hormônios (Boleli et al., 2002). A atuação simultânea dessas células tem por objetivo manter 0 adequado funcionamento do intestino, contudo fatores patogênicos e componentes presentes no alimento podem romper essa condição (Wallach, 2000). Existem alguns processos envolvidos com a manutenção do tecido intestinal como a proliferação, diferenciação e perda celular, e a condição ideal é quando existe um equilíbrio entre esses processos. No entanto, quando algum fator afeta negativamente essa relação ocorre um aumento da atividade proliferativa para garantir a taxa de renovação do tecido, modificando o epitélio intestinal, podendo acarretar no aumento da profundidade de cripta e alteração no tamanho das vilosidades intestinais (Pluske et al., 1997).

Além de possíveis alterações no tecido intestinal, deve-se considerar algumas interações negativas entre os nutrientes dos alimentos, principalmente quando são utilizados coprodutos na alimentação de aves que possuem qualidade inferior. $A$ grande quantidade de $A G L$ na composição de OAS na dieta pode resultar em uma reação entre o grupo ácido do AGL e os minerais ionizados, como o cálcio, formando sabões insolúveis que tornam os ácidos graxos e minerais indisponíveis para as aves afetando negativamente 0 valor energético da gordura e a retenção de minerais (Bregendahl, 2006).

Uma alternativa possível para minimizar os efeitos deletérios dos AGL seria adicionar lecitina nas dietas dos animais. A lecitina é um emulsificante que têm como principal característica possuir em sua composição compostos com estruturas que apresentam afinidade pela água e lipídios, auxiliando no transporte e na absorção da gordura.

Assim, o presente estudo teve por objetivo avaliar a associação da lecitina de soja com o óleo ácido de soja adicionado em diferentes níveis na dieta e verificar seu efeito sobre a histologia intestinal e qualidade óssea de codornas japonesas.

\section{MATERIAL E MÉTODOS}

Animais e Dietas Experimentais

O experimento foi conduzido no biotério do Laboratório de Ensino e Experimentação Zootécnica Prof. Dr. Renato Rodrigues Peixoto - UFPEL. Um 
entre força e distância (distância que a sonda percorre até romper o osso). A flexibilidade representa a deformação do osso em função da força aplicada sobre ele.

As tíbias direitas foram utilizadas para a determinação da matéria mineral de acordo com a metodologia da AOAC (1995).

\section{Análise Estatística}

O modelo matemático utilizado para análise estatística foi: Yijk $=\mu+\mathrm{Oi}$ $+\mathrm{Nj}+\mathrm{Ek}+(\mathrm{UM}) \mathrm{ijk}+$ eijk, onde: $\mu=$ média geral; $\mathrm{Oi}=$ efeito do tipo de óleo $(\mathrm{i}$ $=1,2) ; \mathrm{Nj}=$ efeito dos níveis de suplementação com óleo $(j=1,2)$; Ek = Efeito da Lecitina $(\mathrm{k}=1,2)$; ONEijk = efeito da interação ONE no nível i, j, k; e eijk = erro aleatório.

Os dados foram analisados por ANOVA com $\mathrm{O}, \mathrm{N}$ e $\mathrm{E}$ como fatores fixos usando o pacote estatístico $R$ ( $R$ Core Team, 2015). O valor de probabilidade de $p<0.05$ foi considerado estatisticamente significativo.

\section{RESULTADOS}

\section{Morfometria intestinal}

Os resultados das variáveis de morfometria intestinal do duodeno estão apresentados na Tabela 2. Para profundidade de cripta foi verificada a interação dupla significativa entre os fatores tipo de óleo e lecitina $(P=0,04)$. $\mathrm{Na}$ Figura 1 é possível observar que as codornas alimentadas com dietas contendo OAS na presença de LEC apresentaram significativamente menor profundidade de cripta em relação as aves alimentas apenas com OAS. No entanto, quando as aves foram alimentadas com ODS essas diferenças devido a presença de LEC não foram significativas.

$\mathrm{Na}$ avaliação de altura de vilosidade, não foram observadas interações significativas entre os fatores estudados. A inclusão de alto nível de óleo na dieta (8\%) não afetou significativamente a altura das vilosidades em comparação com um nível intermediário de óleo (4\%). De forma semelhante a inclusão de $1 \%$ de LEC na dieta não influenciou a altura de vilosidades. No entanto, as codornas alimentadas com óleo ácido apresentaram menor altura de vilos em comparação com as aves alimentadas com óleo degomado de soja $(P=0,04)$.

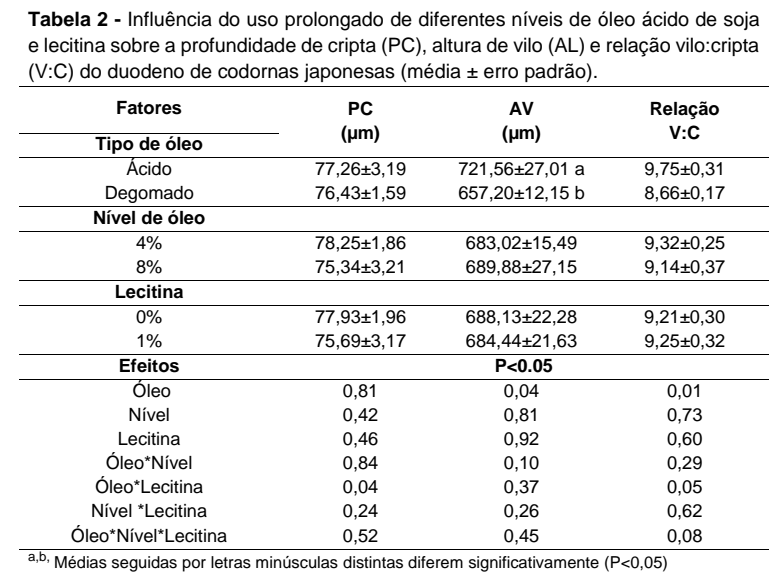

a,b, Médias seguidas por letras minúsculas distintas diferem significativamente $(P<0,05)$

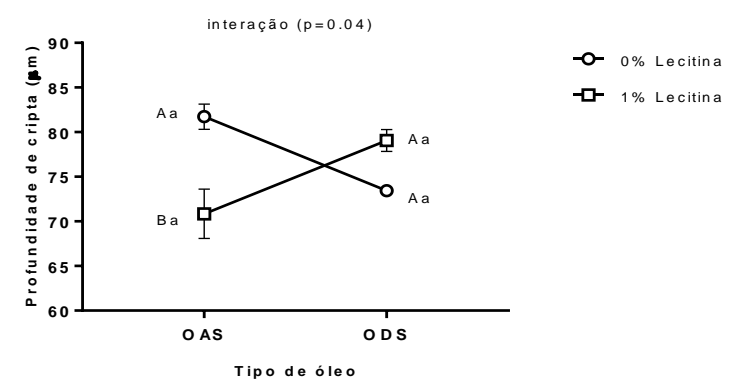

Médias seguidas por letras minúsculas distintas são significativamente diferentes entre tipos de óleos; Médias seguidas por letras maiúsculas distintas diferem significativamente entre presença e ausência de lecitina.

Figura 1 - Efeito da interação entre tipo de óleo e a presença e ausência de lecitina sobre a profundidade de cripta (duodeno) de codornas japonesas.

Conforme pode ser observado na Figura 2 houve uma interação dupla significativa entre o tipo de óleo e o uso 
de lecitina sobre a relação vilo:cripta $(\mathrm{P}=0,05)$. $\mathrm{Na}$ presença de suplementação com LEC as codornas que se alimentaram com ODS apresentaram significativamente menor relação vilo:cripta em comparação com aquelas alimentadas com OAS. No entanto, na ausência de suplementação com LEC não foram encontradas diferenças significativas para a relação vilo:cripta entre aves alimentadas com ODS ou OAS.

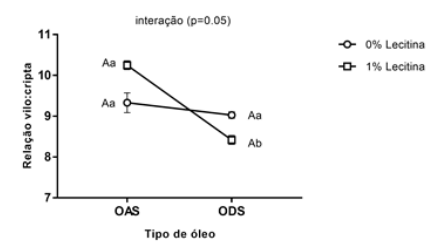

Médias seguidas por letras minúsculas distintas diferem significativamente entre tipos de óleos: Médias seguidas por letras maiúsculas distintas diferem significativamente entre presença e ausência de
lecitina.

Figura 2 - Efeito da interação entre o tipo de óleo e a presença e ausência de lecitina sobre a relação vilo:cripta (duodeno) de codornas japonesas.

\section{Qualidade óssea}

Não foram observadas interações significativas entre os níveis dos fatores estudados para todas as características ósseas analisadas (Tabela 3). Também não foram encontradas diferenças significativas entre os tipos e níveis de inclusão de óleo ou efeito da suplementação com LEC, exceto para o conteúdo mineral das tíbias das codornas que foi maior nas aves alimentadas com $4 \%$ de óleo em comparação com $8 \%$ de óleo $(P<0,01)$. As aves suplementadas com LEC apresentaram menor porcentagem de cinzas em comparação com as aves não suplementadas $(\mathrm{P}<0,0001)$.

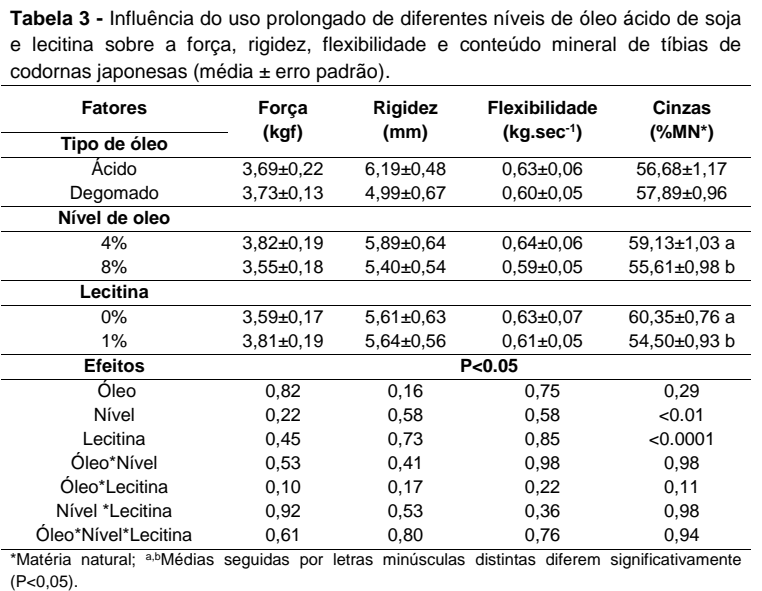

\section{DISCUSSÃO}

Os lipídios são extremamente importantes na dieta de aves, além de complementar a energia das dietas, conferem outros diversos benefícios, dentre eles podemos citar a melhora da digestibilidade dos nutrientes pela redução da taxa de passagem do alimento (Beterchini, 2006), acarretando num maior tempo de exposição do alimento com as enzimas digestivas e em contato com o epitélio intestinal. Contudo, a qualidade dessa fonte energética influencia diretamente 0 aproveitamento dos nutrientes, afetando o desenvolvimento e manutenção do tecido intestinal, sendo esse local responsável pela digestão final dos alimentos e absorção.

Alguns parâmetros podem ser utilizados para avaliar a saúde do epitélio intestinal, dentre eles temos a profundidade de cripta. Essa avalição nos fornece um indicativo da atividade proliferativa celular, e o aumento dessa medida está relacionado com o acréscimo da taxa de renovação tecidual, pela perda de epitélio das extremidades das vilosidades (Bolel et al., 2002).

O OAS demonstrou exercer efeito negativo sobre a profundidade de cripta, já que aumentou esse resultado. Este fato pode estar relacionado possivelmente com o reduzido aproveitamento dos AGL presentes em grandes quantidades no OAS, já que no 
mínimo $50 \%$ dos lipídeos são absorvidos na forma de 2monoacilglicerol (Leeson e Summers, 2005). Assim, os AGL não absorvidos tornam-se agentes agressores do epitélio intestinal, resultando na abrasão das vilosidades e consequentemente na perda de tecido, acarretando no aumento da taxa de renovação do epitélio.

Ao adicionar a lecitina nas dietas com OAS pode ter ocorrido um aumento na incorporação dos ácidos graxos não polares dentro da fase micelar (Øverland et al., 1994), aumentando a digestão e absorção desses compostos (Bellaver e Snizek, 1999), reduzindo a sua quantidade na forma 'livre' e seus efeitos negativos sobre 0 epitélio intestinal.

$\mathrm{Na}$ literatura é encontrada uma variabilidade muito grande na composição nutricional e qualidade (quantidade de AGL) dos óleos ácidos de soja. Isso deve-se principalmente pela variação dos processos de refino do óleo e também pela oscilação na composição dos grãos de soja. No presente estudo, o resultado $64,7 \%$ para o valor de acidez em ácido oleico, demonstra que o óleo ácido apresentava melhor qualidade do que óleos ácidos testados em outros estudos, como no de Peña et al. (2014) e Raber et al. (2009), que encontraram 77,3 e $79 \%$ de AGL nos OAS, respectivamente. Esse fato pode explicar os menores efeitos deletérios sobre a altura das vilosidades do duodeno das codornas alimentadas com OAS em comparação com as aves que receberam dietas com o ODS.

A altura de vilo está relacionada com a taxa de proliferação celular, que ocorre na sua maioria na região da cripta e diminui gradativamente conforme se direciona para a região apical dos vilos (Boleli et al., 2002), contudo ela ocorre em diversos pontos do epitélio. A proliferação nas criptas corresponde a $55 \%$, enquanto que a região média é responsável por $32 \%$ e a região apical por apenas $8 \%$ das divisões mitóticas (Applegate et al., 1999). Com essas informações é possível indicar que a relação altura de vilo e profundidade de cripta pode ser utilizada como um parâmetro da saúde intestinal, em que vilosidades altas e criptas rasas resultam numa maior relação $\mathrm{V}: \mathrm{C}$, indicando uma melhor absorção de nutrientes e menores perdas com a renovação celular (Arruda et al., 2008). Entretanto, um fato importante a considerar é que aproximadamente $50 \%$ da proliferação celular intestinal pode ocorrer ao longo dos vilos (Boleli et al., 2002).

O ODS é a fonte lipídica mais utilizada na dieta de frangos de corte (Silva, 2015), devido sua alta qualidade e disponibilidade. O conteúdo de AGL encontrado nesse óleo não ultrapassa $1 \%$, e no presente estudo foi encontrado um índice de acidez em ácido oleico de apenas $0,28 \%$. A lecitina de soja foi adicionada nas dietas com o objetivo de auxiliar no aproveitamento dos AGL, contudo esses compostos estão pouco presente no ODS. Esse fato pode ter contribuído com a redução da relação $\mathrm{V}: \mathrm{C}$ em animais alimentados com ODS+LEC, já que um excesso de nutrientes pode ter acarretado na redução da absorção, resultando na lesão do epitélio (perda de células), pela presença de compostos na luz intestinal ocasionando uma maior taxa de renovação celular (proliferação celular).

Muitos nutrientes presentes na dieta de aves influenciam diretamente 0 crescimento e a manutenção óssea, contudo para ocorrer o efeito benéfico desses compostos é necessário que eles estejam disponíveis para a absorção pelas aves. Fatores físicoquímicos podem influenciar na biodisponibilidade dos minerais, assim 
sendo ao introduzir um ingrediente na dieta de aves, deve-se conhecer sua composição já que pode ocorrer antagonismo entre nutrientes e afetar adversamente seu aproveitamento (Pascoal e Fonseca, 2014).

Codornas japonesas são aves especializadas na produção de ovos, e para esses animais é imprescindível se preocupar com o aproveitamento dos minerais da dieta, já que existe a formação da casca dos ovos. Essa porção do ovo demanda em torno de $10 \%$ do cálcio total estocado no organismo da ave (Carvalho et al., 2014), e na fase de produção, esse mineral fica armazenado no osso medular, que funciona como um reservatório utilizado como fonte secundária de cálcio para a formação da casca (Whitehead, 2004).

A qualidade óssea pode ser avaliada de diversas formas, dentre elas temos a: força, rigidez e flexibilidade. A força óssea é dada pela força aplicada por área óssea e é influenciada por fatores como tamanho ósseo e composição mineral. A razão entre a resistência e a distância do osso (tamanho do osso) representa rigidez óssea $(\mathrm{mm})$. A flexibilidade óssea é representada pela área, e mede a deformidade óssea em função da força aplicada sobre o mesmo (Gopinger et al., 2017). Outro parâmetro passível de ser relacionado com a qualidade óssea é o conteúdo mineral, já que ele corresponde a $70 \%$ da composição total do osso (Júnior, 2002).

Uma das hipóteses do presente estudo era confirmar a redução da qualidade óssea nas aves alimentadas com as dietas contendo apenas o OAS, porém para as variáveis de força, rigidez e flexibilidade nenhum efeito foi observado. Ao avaliar a quantidade total de minerais das tíbias das codornas os fatores nível do óleo e lecitina atuaram sobre a resposta.
Segundo Rostagno et al. (2017) o nível prático indicado para o uso de fontes lipídicas na dieta de poedeiras é de $4 \%$, e o nível máximo é de $7 \%$. 0 uso das gorduras na dieta de aves pode trazer diversos beneficios, contudo o excesso dessa fonte acarreta em diversos efeitos adversos, como aumento da taxa de passagem do alimento pelo trato gastrointestinal acarretando na redução do aproveitamento dos nutrientes das dietas, além de aumentar a quantidade de excretas no ambiente (Macari et al., 2002). Outra questão a ser considerada ao utilizar lipidios nas dietas de aves é o seu perfil de ácidos graxos, e ao se tratar da qualidade óssea os ácidos graxos poliinsaturados (AGPI) estão intimamente relacionados com a deposição e reabsorção óssea (Liu e Denbow, 2001).

O ácido linoleico (ômega 6) é convertido em ácido araquidonico e estocado nas membranas das células do tecido ósseo (osteoblastos e condrócitos), e diversos fatores podem estimular a liberação do ácido araquidônico que vai servir de substrato para a síntese de prostaglandinas (Watkins et al., 2001). Essas substâncias em altas concentrações inibem a formação da matriz óssea, enquanto baixas concentrações podem estimular a formação do osso (Liu e Denbow, 2001). Outro AGPI que tem grande importancia na formação óssea é o ácido linolênico ( $\triangle-3$ ), ele atua reduzindo a incorporação do ácido araquidonico nas membranas, assim afetando na diminuição das prostaglandinas (Calder, 1998).

Desta forma, ao incluir menor quantidade de óleo nas dietas pode ter proporcionado um fornecimento mais adequado dos ácidos graxos que atuam na formação e reabsorção óssea, e o uso de $8 \%$ de lipídios pode ter acarretado num desbalanço desses nutrientes aumentando a reabsorção 
ósssea e assim diminuindo a quantidade total de minerais. O mesmo principio pode ser utilizado para explicar o maior teor de cinzas nas tíbias de codornas alimentadas com dietas sem LEC.

A lecitina crua de soja é uma mescla de $60-65 \%$ de gomas (fosfolipídios) e 40-35\% de óleo residual (Blas et al., 2010), e o perfil de ácidos graxos se aproxima muito da encontrada no óleo de soja. Esse fato pode ter contribuído com a redução da quantidade de mineral nas tíbias de codornas alimentadas com dietas contendo o emulsificante, pois ele pode ter contribuido com quantidades de ácidos graxos envolvidos no metabolismo ósseo, acarretando no desequilibrio deles e ocasionando o aumento da taxa de reabsorção óssea.

Apesar dos resultados da histologia intestinal demonstrarem que a associação da lecitina com o OAS melhorou os parâmetros de profundidade de cripta e relação $\mathrm{V}: \mathrm{C}$, a significância de alguns fatores isolados como tipo de óleo para altura de vilo e do nível e lecitina para o conteúdo mineral das tíbias dificulta traçar um comportamento padrão entre os fatores de estudo.

\section{CONCLUSÃO}

A adição de lecitina de soja nas dietas contendo óleo ácido de soja foi positiva, reduzindo a profundidade de cripta e aumentando a relação vilo:cripta. Contudo, não foi verificado nenhum efeito da associação do emulsificante sobre a qualidade óssea das aves. O nível mais alto de óleo (8\%) na dieta e a lecitina (1\%) atuaram de forma isolada sobre a resposta de minerais totais das tíbias das aves, reduzindo essa variável.

\section{Agradecimentos}

À Coordenação de Aperfeiçoamento de Pessoal de Nível
Superior (CAPES) pela concessão da bolsa de mestrado. À Fundação de Amparo à Pesquisa do Estado do Rio Grande do Sul (FAPERGS) e ao Conselho Nacional de Desenvolvimento Científico e Tecnológico (CNPq) pelo financiamento do projeto através dos Editais 02/2014 - PqG e 14/2014 $\mathrm{MCTI} / \mathrm{CNPq}$, respectivamente. À Empresa Sulina Óleos pelo fornecimento dos óleos ácido, degomado e a lecitina de soja, utilizados nesta pesquisa.

\section{Notas Informativas}

Os métodos e protocolos utilizados neste experimento foram aprovados pela Comissão de Ética em Experimentação Animal (CEEA) da Universidade Federal de Pelotas, sob o protocolo número 3772.

\section{REFERÊNCIAS}

BETERCHINI, A. G. Nutrição de monogástricos. UFLA, Lavras, 2006. $301 p$.

ASSOCIATION OF OFFICIAL ANALYTICAL CHEMISTRY - AOAC. Official methods of analysis. $16 \mathrm{ed}$. Arlington: AOAC International, 1995. APPLEGATE, T. J.; DIBNER, J. J.; KITCHELL, M. L. et al. Effect of turkey (Meleagridis gallopavo) breeder hen age and egg size on poult development.

2. Intestinal villus growth, enterocyte migration and proliferation of the turkey poult. Comp. Biochem. Physiol. Part B. Biochemestry \& Molecular Biology Journal,124:381-389, 1999.

ARRUDA, M. V. A.; FERNANDES, R. T. V.; SILVA, J. M. et al. Avaliação morfo histológica da mucosa intestinal de coelhos alimentados com diferentes níveis e fontes de fibra. Revista Caatinga, 21:01-11, 2008.

BELLAVER, C.; SNIZEK, P. N. J. Processamento da soja e suas implicações na alimentação de suínos e 
aves. In: Congresso Brasileiro da Soja, 1999, Londrina, p. 183-199, 1999.

BOLELI, I. C.; MAIORKA, A.; MACARI, M. Estrututa Funcional do Trato Digestório In: MACARI, M.; FURLAN, R.; GONZALES. Fisiologia. Aviária Aplicada à Frangos de Corte. $2^{\circ}$ ed. São Paulo FUNEP/UNESP, 2002, 143$148 p$.

BRAGA, J.P.; BAIÃO, N. C. Suplementação lipídica no desempenho de aves em altas temperaturas. Cadernos Técnicos de Veterinária e Zootecnia, 31:23-28, 2001.

BREGENDAHL, K. Free fatty acids in diet for laying hens. Feed energy topic: laying hen diets. Department of Animal Science - State University - lowa, 2006.

BLAS, C.; MATEOS, G. G.; GRACÍAREBOLLAR, P. Tablas de composición y valores nutritivos de alimentos para la fabricación de piensos compuestos. 3 ed. Fundación Española para el Desarrollo de la Nutrición Animal. Madrid, Espanha, 2010, 502 p.

CALDER, P. C. Immunoregulatory and anti-inflammatory effects of $n-3$ polyunsaturated fatty acids. Brazilian Journal of Medical and Biological

Research, 31:467-490, 1998.

MAIA, R. C.; TAVERNATI, F. C.; ALBINO, L. F. et al. Formação e Qualidade dos Ovos. In: CARVALHO, R. C. M.; TAVERNARI, F. C.; ALBINO, L. F. T et al. Galinhas Poederias: Criação e Alimentação. $1^{\circ}$ ed, 2014, 276 - 314 p.

CHERRY,J.P.;KRAMER, W. Lecithins: sources, manufactures \& uses. In: Plant sources of lecithin, capítulo 3. The American Oil Chemist's Society. Champaing, Ilinois, 1989, 16-31p.

FIRMAN, D. J.; KAMYAB, A.; LEIGH, H. Comparison of Fat Sources in Rations of Broilers from Hatch to Market. International Journal Poultry Science, 7:1152 1155, 2008.

GOPINGER, E.; KRABBE, E. L.; SUREK, D. L. et al. Live Performance,
Carcass, and Bone Quality Responses of Grower and Finisher Broilers to Dietary Metabolizable Energy Levels. Revista Brasileira de Ciência Avícola, 19:559-566, 2017.

JÚNIOR, J. M. P. Estrutura e função do tecido ósseo. In: MACARI, M.; FURLAN, R.; GONZALES. Fisiologia. Aviária Aplicada à Frangos de Corte. $2^{\circ}$ ed. São Paulo FUNEP/UNESP, 2002, 375 p.

LEESON, S.; SUMMERS, J. D. Commercial Poultry Nutrition. $3^{\circ}$ ed. Nottingham University Press Manor Farm, Church Lane, Thrumpton, Nottingham, England, 2005.

LIU, D.; DENBOW, D. M. Maternal Dietary Lipids Modify Composition of Bone Lipids and Ex Vivo Prostaglandin E2 Production in Early Postnatal Japanese Quail. Poultry Science, 80:1344-1352, 2001.

MACARI, M.; FURLAN, R. L.; GONZALES, E. Fisiologia Aviária Aplicada à Frangos de Corte. (AJ editora FUNEP/UNESP). 2 ed. Jaboticabal, 2002.

MATEOS, G.G.; SALDAÑA B.; FRIKHA, $M$. et al. Aceites resultantes de procesos industriales en piensos para monogástricos: Oleinas, aceites reconstituidos y lectinas. In: Curso de especialization FEDNA. Madrid, 7 y 8 de noviembro de 2012. 2012. 33-49 p.

ØVERLAND, M.; MROZ, Z.; SUNDST $\varnothing L, F$. Effect of lecithin on the apparent ileal and overall digestibility of crude fat and fatty acids in pigs. Journal Animal Science, 72:2022-2028, 1994. PASCOAL, J. H. V.; FONSECA, L. A. Função e Disponibilidade dos Minerais. SAKOMURA, N. K.; SILVA, J. H. V.; COSTA F. G. P. et al. In: Nutrição de Não Ruminates. $1^{\circ}$ ed. São Paulo FUNEP/UNESP, Jaboticabal, Brasil, 2014, 129-141p.

PEÑA, J.E.M.; VIEIRA, S. L.; BORSATTI, L. et al. Soybean Oil Industry by Broiler Chickens: Acidulated Soapstock , Lecithin , Glycerol and 
Their. Revista Brasileira de Ciência Avícola, 16:437-442, 2014.

PLUSKE, J. R.; HAMPSON, D. J.; WILLIAMS, I. H. Factors influencing the structure and function of the small intestine in the weaned pig: a review. Livestock Production Science, 51:215-236, 1997.

$R$ CORE TEAM. R A Lang. Environ. Stat. Comput. R Found. Stat. Comput., Vienna, Austria. ISBN 3-900051-07-0, URL http//www.R-project.org/. 55:275286, 2015.

RABER, M.; RIBEIRO, A.; KESSLER, A. et al. Suplementação de glicerol ou de lecitina em diferentes níveis de ácidos graxos livres em dietas para frangos de corte. Ciência Animal Brasiliera, 10:745-753, 2009.

ROSTAGNO, H. S.; ALBINO, L. F. T.; HANNAS, M. I. et al. Tabelas Brasileiras Para Aves e Suínos: Composição dos Alimentos e Exigências Nutricionais. 4 ed. Viçosa: Departamento de Zootecnia, UFV, 2017, 488p.

SILVA, L. M. Avaliação energética do óleo ácido de soja, combinado com glicerol e lecitina, e o uso em rações para frangos de corte. 2015. Viçosa, 100f, Dissertação (Mestrado em Zootecnia) - Programa de PósGraduação em Zootecnia da Universidade Federal de Viçosa.

WALLACH, M. The control of cocciodiosis in chickens by maternal immunization. In: Workshop at the national veterinary institute: coccidiose and clostridial

injections in broiler chickens. $A B I C$ Ltda, Jerusalém, Israel, 2000. 1-3 p.

WATKINS, B. A.; LI, Y.; SEIFERT, M. F. Lipids as modulators of bone remodelling. Current Opinion in Clinical Nutrition \& Metabolic Care. 4:105-110, 2001.

WHITEHEAD, C. C. 2004. Overview of bone biology in the egg-laying hen.

Poultry Science. 83:193-199. 